\title{
Epidemiological and PK/PD cutoff values determination and PK/PD-based dose assessment of gamithromycin against Haemophilus parasuis in piglets
}

Yu-Feng Zhou ${ }^{1,2+}$, Ming-Xiao Bu $u^{1,2 \dagger}$, Ping Liu ${ }^{1,2}$, Jian Sun ${ }^{1,2}$, Ya-Hong Liu ${ }^{1,2}$ and Xiao-Ping Liao ${ }^{1,2,3^{*}}$

\begin{abstract}
Background: Gamithromycin is a macrolide approved for the treatment of bovine and swine respiratory diseases. Our study aims to establish the clinical breakpoint and optimum dose regimen for gamithromycin against Haemophilus parasuis in piglets.

Results: Gamithromycin was well absorbed and fully bioavailable (87.2-101\%) after intramuscular and subcutaneous administrations. The MICs of gamithromycin for 192 clinical H. parasuis isolates ranged from 0.008 to $128 \mathrm{mg} / \mathrm{L}$ and the epidemiological cutoff (ECOFF) was calculated as $1.0 \mathrm{mg} / \mathrm{L}$. A large potentiation effect of serum on in vitro susceptibility of gamithromycin was observed for $\mathrm{H}$. parasuis, with broth/serum ratios of 8.93 for MICs and 4.46 for MBCs, respectively. The postantibiotic effects were $1.5 \mathrm{~h}(1 \times \mathrm{MIC})$ and $2.4 \mathrm{~h}(4 \times \mathrm{MIC})$, and the postantibiotic sub-MIC effects ranged from 2.7 to $4.3 \mathrm{~h}$. Gamithromycin had rapid and concentration-dependent killing against $H$. parasuis, and the $A \cup C_{24 h} / M I C$ ratio correlated well with ex vivo efficacy $\left(R^{2}=0.97\right)$. The $A \cup C_{24 h} / M I C$ targets in serum associated with bacteriostatic, bactericidal and eradication activities were 15.8, 30.3 and 41.2, respectively. The PK/PD-based population dose prediction indicated a probability of target attainment (PTA) for the current marketed dose $(6 \mathrm{mg} / \mathrm{kg})$ of $88.9 \%$ against $\mathrm{H}$. parasuis. The calculated gamithromycin dose for a PTA $\geq 90 \%$ was $6.55 \mathrm{mg} / \mathrm{kg}$. Based on Monte Carlo simulations, the PK/PD cutoff (COPD) was determined to be $0.25 \mathrm{mg} / \mathrm{L}$.
\end{abstract}

Conclusion: The determined cutoffs and PK/PD-based dose prediction will be of great importance in gamithromycin resistance surveillance and serve as an important step in the establishment of optimum dose regimen and clinical breakpoints.

Keywords: Cutoff, Gamithromycin, H. Parasuis, PK/PD, Piglet

* Correspondence: xpliao@scau.edu.cn

${ }^{\dagger}$ Yu-Feng Zhou and Ming-Xiao Bu contributed equally to this work.

${ }^{1}$ National Risk Assessment Laboratory for Antimicrobial Resistance of Animal Original Bacteria, College of Veterinary Medicine, South China Agricultural University, Guangzhou, China

${ }^{2}$ Guangdong Provincial Key Laboratory of Veterinary Pharmaceutics Development and Safety Evaluation, South China Agricultural University, Guangzhou, China

Full list of author information is available at the end of the article

\section{Background}

Haemophilus parasuis is the etiological agent of Glässer's disease that causes inflammatory infections such as fibrinous polyserositis, meningitis, arthritis and bronchopneumonia in weaned piglets [1]. As a commensal pathogen in porcine respiratory tract, $H$. parasuis is frequently associated with porcine reproductive and respiratory syndrome virus, porcine circovirus type 2 and Streptococcus suis infections, resulting in large economic losses in the swine

(c) The Author(s). 2020 Open Access This article is licensed under a Creative Commons Attribution 4.0 International License, which permits use, sharing, adaptation, distribution and reproduction in any medium or format, as long as you give appropriate credit to the original author(s) and the source, provide a link to the Creative Commons licence, and indicate if changes were made. The images or other third party material in this article are included in the article's Creative Commons licence, unless indicated otherwise in a credit line to the material. If material is not included in the article's Creative Commons licence and your intended use is not permitted by statutory regulation or exceeds the permitted use, you will need to obtain permission directly from the copyright holder. To view a copy of this licence, visit http://creativecommons.org/licenses/by/4.0/. The Creative Commons Public Domain Dedication waiver (http://creativecommons.org/publicdomain/zero/1.0/) applies to the data made available in this article, unless otherwise stated in a credit line to the data. 
industry worldwide $[2,3]$. There are currently 15 serotypes of $H$. parasuis and serotypes 1,5 and 10 are the most virulent causing rapid death of infected piglets $[4,5]$. The vaccines currently available only provide partial protection due to the serotype diversity and this is problematic because many strains cannot be serotyped [6]. Thus, antibiotic therapy still represents the most effective strategy for controlling the spread of $H$. parasuis infections.

Gamithromycin is a macrolide of the azalide subclass that approved for the treatment of bovine and swine respiratory diseases $[7,8]$. In naturally occurring bovine respiratory diseases associated with Mannheimia haemolytica and Pasteurella multocida, the area under the concentration-time curve to minimal inhibitory concentration ratio (AUC/MIC) was the pharmacokinetic/pharmacodynamic (PK/PD) index that best predicted the efficacy of gamithromycin [9]. However, PK/PD data for gamithromycin against swine respiratory pathogens including $H$. parasuis have not been pursued. In addition, previous susceptibility study for gamithromycin against H. parasuis resulted in $\mathrm{MIC}_{50}$ and $\mathrm{MIC}_{90}$ values of 0.25 and 0.5 (range 0.06-4) $\mathrm{mg} / \mathrm{L}$, respectively [7]. However, as the crucial interpretative criteria to categorize the results of antimicrobial susceptibility testing (AST), the clinical breakpoints (CBPs) of gamithromycin for the relevant bacterial target pathogens still remain unclear.

In this study, we described ex vivo PK/PD relationships of gamithromycin in porcine serum against $H$. parasuis and estimated the magnitude of PK/PD parameter to achieve required efficacies. Our studies were designed to (i) determine the serum matrix effect on susceptibility, post-antibiotic effect (PAE) and post-antibiotic sub-MIC effect (PA-SME), (ii) evaluate the probability of target attainment (PTA) of the current dose of gamithromycin $(6.0 \mathrm{mg} / \mathrm{kg}$ ) for clinically isolated $H$. parasuis strains, and (iii) establish the relevant MIC cutoff values including epidemiological cutoff value [ECOFF; synonym of wild-type cutoff $\left.\left(\mathrm{CO}_{\mathrm{WT}}\right)\right]$ and $\mathrm{PK} / \mathrm{PD}$ cutoff value $\left(\mathrm{CO}_{\mathrm{PD}}\right.$; named by VetCAST as the PK/PD breakpoint) to assist the selection of a CBP for gamithromycin against $H$. parasuis.

\section{Results}

\section{Gamithromycin PK profiles in serum of piglets}

We observed gamithromycin peak levels in piglets $\left(\mathrm{C}_{\max }\right.$; 0.99 and $0.61 \mathrm{mg} / \mathrm{L}$ ) at 0.36 and $1.59 \mathrm{~h}$ after intramuscular (IM) and subcutaneous (SC) routes, respectively (Table 1). Following a single IM injection of gamithromycin at 6.0 $\mathrm{mg} / \mathrm{kg}$, the serum drug levels were $>0.10 \mathrm{mg} / \mathrm{L}$ up to $12 \mathrm{~h}$ and still detectable at $96 \mathrm{~h}$ (Fig. 1). The serum terminal half-life $\left(\mathrm{T}_{1 / 2}\right)$ ranged from 25.4 to $29.4 \mathrm{~h}$ after IV, IM and $\mathrm{SC}$ administrations of gamithromycin. Piglets received the drug IM and SC resulted in $\mathrm{AUC}_{0-\infty}$ of 6.63 and $5.72 \mathrm{mg} \cdot \mathrm{h} /$ $\mathrm{L}$ that were comparable to the IV $\mathrm{AUC}_{0-\infty}$ at the same dose.
Table 1 PK parameters of gamithromycin in serum from six piglets following single IV, IM and SC administrations at $6.0 \mathrm{mg} /$ $\mathrm{kg}(n=6)$

\begin{tabular}{lllll}
\hline PK parameters & Unit & IV route & IM route & SC route \\
\hline$T_{\max }$ & $\mathrm{h}$ & - & $0.36 \pm 0.24$ & $1.59 \pm 1.40$ \\
$\mathrm{C}_{\max }$ & $\mathrm{mg} / \mathrm{L}$ & - & $0.99 \pm 0.29$ & $0.61 \pm 0.14$ \\
$\mathrm{~T}_{1 / 2}$ & $\mathrm{~h}$ & $25.4 \pm 2.90$ & $29.4 \pm 3.71$ & $29.0 \pm 3.44$ \\
$\mathrm{AUC}_{\text {last }}$ & $\mathrm{mg} \cdot \mathrm{h} / \mathrm{L}$ & $6.32 \pm 1.24$ & $6.28 \pm 1.56$ & $5.45 \pm 1.56$ \\
$\mathrm{AUC} \mathrm{C}_{0-\infty}$ & $\mathrm{mg} \cdot \mathrm{h} / \mathrm{L}$ & $6.56 \pm 1.31$ & $6.63 \pm 1.89$ & $5.72 \pm 1.67$ \\
$\mathrm{Cl}$ & $\mathrm{L} / \mathrm{kg} / \mathrm{h}$ & $0.92 \pm 0.19$ & - & - \\
$\mathrm{Cl} / \mathrm{F}$ & $\mathrm{L} / \mathrm{kg} / \mathrm{h}$ & - & $0.91 \pm 0.26$ & $1.05 \pm 0.30$ \\
$\mathrm{~V}_{\mathrm{ss}}$ & $\mathrm{L} / \mathrm{kg}$ & $18.1 \pm 3.20$ & - & - \\
$\mathrm{F}$ & $\%$ & - & 101.1 & 87.2 \\
\hline
\end{tabular}

$T_{\max }$ time to reach peak concentration $\left(C_{\max }\right), T_{1 / 2}$ terminal half-life, $A U C$ the area under the concentration-time curve from 0 to the last sampling point $\left(A \cup C_{\text {last }}\right.$ ) or from 0 to $\infty\left(A \cup C_{0-\infty}\right), C l$ body clearance, $\mathrm{Cl} / \mathrm{F}$ clearance scaled by bioavailability, $V_{s s}$ volume of distribution at steady-state, $F$ bioavailability

This indicated a high bioavailability (87.2-101.1\%) for SC and IM injections of gamithromycin (Table 1).

\section{In vitro susceptibility testing, ECOFF and PAE determinations}

The calculated gamithromycin MIC for $H$. parasuis LM15 in Haemophilus test medium (HTM) broth $(0.25$ $\mathrm{mg} / \mathrm{L})$ was 8 -fold greater than that in serum $(0.031 \mathrm{mg} /$ L), indicating a large potentiation effect by serum. To further confirm the serum effects, MICs and MBCs of gamithromycin were determined in both HTM and serum against 19 selected $H$. parasuis isolates. Interestingly, the geometric means of the MICs and MBCs were significantly different between HTM and serum, resulting in HTM/serum ratios of 8.93 for MICs and 4.46 for MBCs $(P<0.01$; Table 2$)$. For both test matrices, the $\mathrm{MBC} / \mathrm{MIC}$ ratios were relatively low and ranged from 1.61 to 3.21 (Table 2).

The MICs of gamithromycin against our 192 clinical H. parasuis isolates ranged from 0.008 to $128 \mathrm{mg} / \mathrm{L}$ in HTM broth, with $\mathrm{MIC}_{50}$ and $\mathrm{MIC}_{90}$ of 0.125 and $2 \mathrm{mg} /$ L, respectively. The standard goodness-of-fit tests showed that the raw MIC distribution did not match a normal distribution due to the bimodal distribution observed at the MICs of 0.125 and $8 \mathrm{mg} / \mathrm{L}$, respectively. The $17 \mathrm{H}$. parasuis isolates with MICs $>8 \mathrm{mg} / \mathrm{L}$ were therefore removed to obtain the best fitted unimodal MIC distribution [ $\log _{2}$ mean (-3.84), $\log _{2}$ SD (1.73), $n=175$ ] using the Kolmogorov-Smirnov (K-S) normality test at $P>0.1$. The fitted MIC distribution contained $>$ 95\% that possessed gamithromycin MICs between 0.008 and $1 \mathrm{mg} / \mathrm{L}$, and the ECOFF value was therefore calculated to be $1 \mathrm{mg} / \mathrm{L}$ (Fig. 2).

The growth of a strain of $H$. parasuis LM15 by $1.0 \log _{10}$ $\mathrm{cfu} / \mathrm{mL}$ in control group occurred at $1.9 \mathrm{~h}$. After removal 


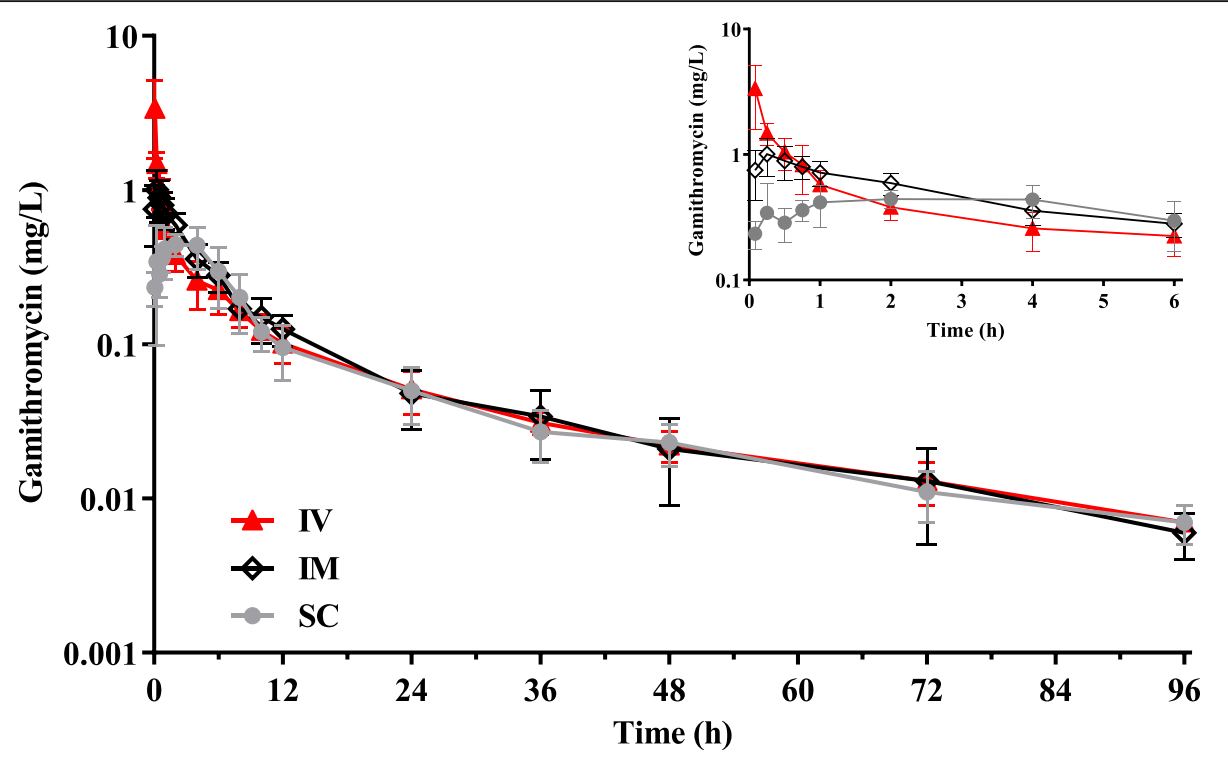

Fig. 1 The concentration-time curves of gamithromycin in porcine serum after single IV, IM and SC administrations at $6.0 \mathrm{mg} / \mathrm{kg}(n=6)$. Inset: the data plotted from 0 to $6 \mathrm{~h}$

of bacterial cells from drug exposure at $1 \times$ and $4 \times$ MIC, regrowth was delayed to 3.4 and $4.3 \mathrm{~h}$ with calculated PAEs ranging from 1.5 to $2.4 \mathrm{~h}$ (Fig. 3a). During sub-MIC phase, as little as 0.1 to $0.3 \times$ MIC gamithromycin produced a further regrowth delay, generating PA-SMEs of 2.7 to $4.3 \mathrm{~h}$ (Fig. 3b).

\section{In vitro and ex vivo antimicrobial activities and PK/PD analysis}

Gamithromycin in vitro time-kill curves using 0.25 to 16 multiples of MIC indicated a concentration-dependent killing pattern. A $0.25 \mathrm{mg} / \mathrm{L}$ drug level (i.e. MIC in HTM) produced a visible growth inhibition whereas > $3.0 \log _{10} \mathrm{cfu} / \mathrm{mL}$ reductions in bacterial density occurred after exposure to gamithromycin at $2 \times \mathrm{MIC}$ for $24 \mathrm{~h}$. At $4 \times$ MIC or higher concentrations, $H$. parasuis densities were reduced by $\sim 4.0 \log _{10} \mathrm{cfu} / \mathrm{mL}$ after only 6 to $9 \mathrm{~h}$ exposure (Fig. 4a).

Serum collected from piglets at $36 \mathrm{~h}$ after IM dosing contained mean drug level of $0.031 \mathrm{mg} / \mathrm{L}$ that equivalent to $\mathrm{MIC}$ in serum, and exerted a visible bactericidal effect

Table 2 Potentiation effect of serum matrix on in vitro susceptibility of gamithromycin against $19 \mathrm{H}$. parasuis isolates $(n=19)^{a}$

\begin{tabular}{llll}
\hline Test matrix & MIC $(\mathrm{mg} / \mathrm{L})$ & $\mathrm{MBC}(\mathrm{mg} / \mathrm{L})$ & $\mathrm{MBC} / \mathrm{MIC}$ ratio \\
\hline HTM & $0.52(0.23)$ & $0.83(0.24)$ & 1.61 \\
Serum & $0.06(0.02)$ & $0.19(0.08)$ & 3.21 \\
HTM/serum ratio $^{b}$ & 8.93 & 4.46 & - \\
\hline
\end{tabular}

${ }^{a} \mathrm{MIC}$ and MBC represent geometric means (SD) using $19 \mathrm{H}$. parasuis isolates ${ }^{b}$ Comparison of Haemophilus test medium (HTM)/serum ratio differences: $P<0.01$
(>2.0 $\log _{10} \mathrm{cfu} / \mathrm{mL}$ ) after $24 \mathrm{~h}$ exposure. Compared with killing pattern in HTM, a more rapid and greater reduction was achieved for serums collected at $12 \mathrm{~h}$ and $24 \mathrm{~h}$ after IM administration that contained the mean drug levels of 0.121 and $0.058 \mathrm{mg} / \mathrm{L}$ (roughly $4 \times$ and $2 \times \mathrm{MIC}$ in serum, respectively). In serum collected up to $12 \mathrm{~h}$ after IM injection, bacterial densities were reduced rapidly by $\sim 4 \log _{10} \mathrm{cfu} / \mathrm{mL}$ after $6 \mathrm{~h}$ exposure (Fig. $4 \mathrm{~b}$ ). There was no further regrowth demonstrating potent ex vivo bactericidal activity of gamithromycin against $H$. parasuis.

The dose-response relationship derived from ex vivo time-kill data were used to calculate the $\mathrm{PK} / \mathrm{PD}$ targets in serum for typical efficacy. The $\mathrm{AUC}_{24 \mathrm{~h}} / \mathrm{MIC}$ index correlated well with ex vivo efficacy $\left(R^{2}=0.97\right)$. The dose-response curve was steep, with the highest decrease from the initial density of $4.68 \log _{10} \mathrm{cfu} / \mathrm{mL}$ (Fig. 5). The target values of $\mathrm{AUC}_{24 \mathrm{~h}} / \mathrm{MIC}$ in serum associated with bacteriostatic, bactericidal and bacterial eradication actions were 15.8, 30.3 and 41.2 , respectively (Table 3).

\section{Dose assessment and PK/PD cutoff calculation}

The dose distribution of gamithromycin covering activity duration of at least 3 days needed a $\mathrm{Cl} / \mathrm{F}$ of $65.5 \mathrm{~L} / \mathrm{kg}$. The scaling factor (SF) obtained by dividing the target value of $\mathrm{AUC}_{24 \mathrm{~h}} / \mathrm{MIC}$ index by $24 \mathrm{~h}$, in our case, the target $\mathrm{AUC}_{24 \mathrm{~h}} / \mathrm{MIC}$ value for a bactericidal effect was 30.3 that equivalent to the serum concentrations over $24 \mathrm{~h}$ of 1.26 multiples of the MIC (i.e. SF = 1.26). The MIC distribution was divided by a scaling factor of 8.93 to bridge HTM and serum. Based on Monte Carlo simulation, the calculated dose of gamithromycin for a PTA $\geq 90 \%$ was $6.55 \mathrm{mg} / \mathrm{kg}$. In view that the current recommended dose 




Fig. 2 The $\log _{2}$-transformed MIC distribution of gamithromycin against clinical $H$. parasuis isolates $(n=192)$ in HTM broth. The bars represent the observed MIC frequencies, and the line represents the predicted frequency based on the best matching normal distribution [Log 2 mean ( 3.84$)$, $\log _{2}$ SD (1.73)]. The ECOFF value represents the epidemiological cutoff value (synonym of wild-type cutoff; COWT)

is $6.0 \mathrm{mg} / \mathrm{kg}$, the corresponding PTA was calculated at 88.9\% (Fig. 6).

Our dose prediction confirmed the adequacy of the current gamithromycin dose for $H$. parasuis infections. The PTAs of the current dose $(6.0 \mathrm{mg} / \mathrm{kg})$ for the typical $\mathrm{AUC}_{24 \mathrm{~h}} / \mathrm{MIC}$ targets at each possible MIC in HTM were thus calculated to obtain the $\mathrm{CO}_{\mathrm{PD}}$ value. At a MIC of $\leq 0.25 \mathrm{mg} / \mathrm{L}$ in HTM, the PTA achieving an $\mathrm{AUC}_{24 \mathrm{~h}} /$ MIC target of 30.3 (for a bactericidal effect) was $96.7 \%$ (Table 4). Therefore, the $\mathrm{CO}_{\mathrm{PD}}$ value of gamithromycin for $H$. parasuis was determined to be $0.25 \mathrm{mg} / \mathrm{L}$.

\section{Discussion}

Gamithromycin is a semisynthetic triamilide antibiotic that disrupts protein synthesis by binding the ribosomal $50 \mathrm{~S}$ subunit and causing premature detachment of polypeptide chains [7]. To our knowledge, this study is the first to address the PK/PD relationships of gamithromycin against $H$. parasuis in piglets. We demonstrated that gamithromycin was absorbed rapidly into the systemic circulation after IM and SC administrations with bioavailabilities between 87.2 and $101 \%$. Following a single IM dose of gamithromycin at $6.0 \mathrm{mg} / \mathrm{kg}$, serum drug concentrations remained above the MIC of $H$. parasuis LM15 beyond $36 \mathrm{~h}$. Despite having limited PAE, gamithromycin has been shown to express an extended lung tissue half-life, which may also support its extended antimicrobial activity $[8,10]$.
Gamithromycin displayed a predominantly bactericidal activity in vitro against major swine respiratory diseases (SRD) pathogens such as $H$. parasuis, $P$. multocida and Actinobacillus pleuropneumoniae [7]. In studies involving field strains isolated in European areas, gamithromycin showed $\mathrm{MIC}_{90}$ values of $0.5,1$ and $4 \mathrm{mg} / \mathrm{L}$ against $H$. parasuis, $P$. multocida and A. pleuropneumoniae, respectively [8]. Similar to other veterinary macrolides such as tulathromycin, clinical field trials have indicated that gamithromycin concentrations in serum can be below the $\mathrm{MIC}_{90}$ for the target SRD pathogens while retaining good clinical efficacy $[7,10]$. It may be due to the significant differences of AST results between serum and broth media $[11,12]$. This resulted in the underestimate of gamithromycin clinical efficacy by measuring MICs in broth medium. In fact, the serum effects on antimicrobial activity of macrolides have been reported for most fastidious respiratory pathogens $[11,13]$. In our study, the MICs of gamithromycin against $H$. parasuis in porcine serum were significantly lower than that in HTM. This difference in susceptibility agreed with the previous findings, in which a similar 4 to 16 -fold reduction in tildipirosin MIC was observed for A. pleuropneumoniae by addition of $50 \%$ serum to broth medium [14]. More recently, our study regarding tulathromycin demonstrated that incorporation of increasing proportion of serum to broth from 25 to $75 \%$ progressively reduced the MIC against $S$. suis [13].

The mechanisms for increased susceptibility of $H$. parasuis to macrolides in serum matrix is likely related 

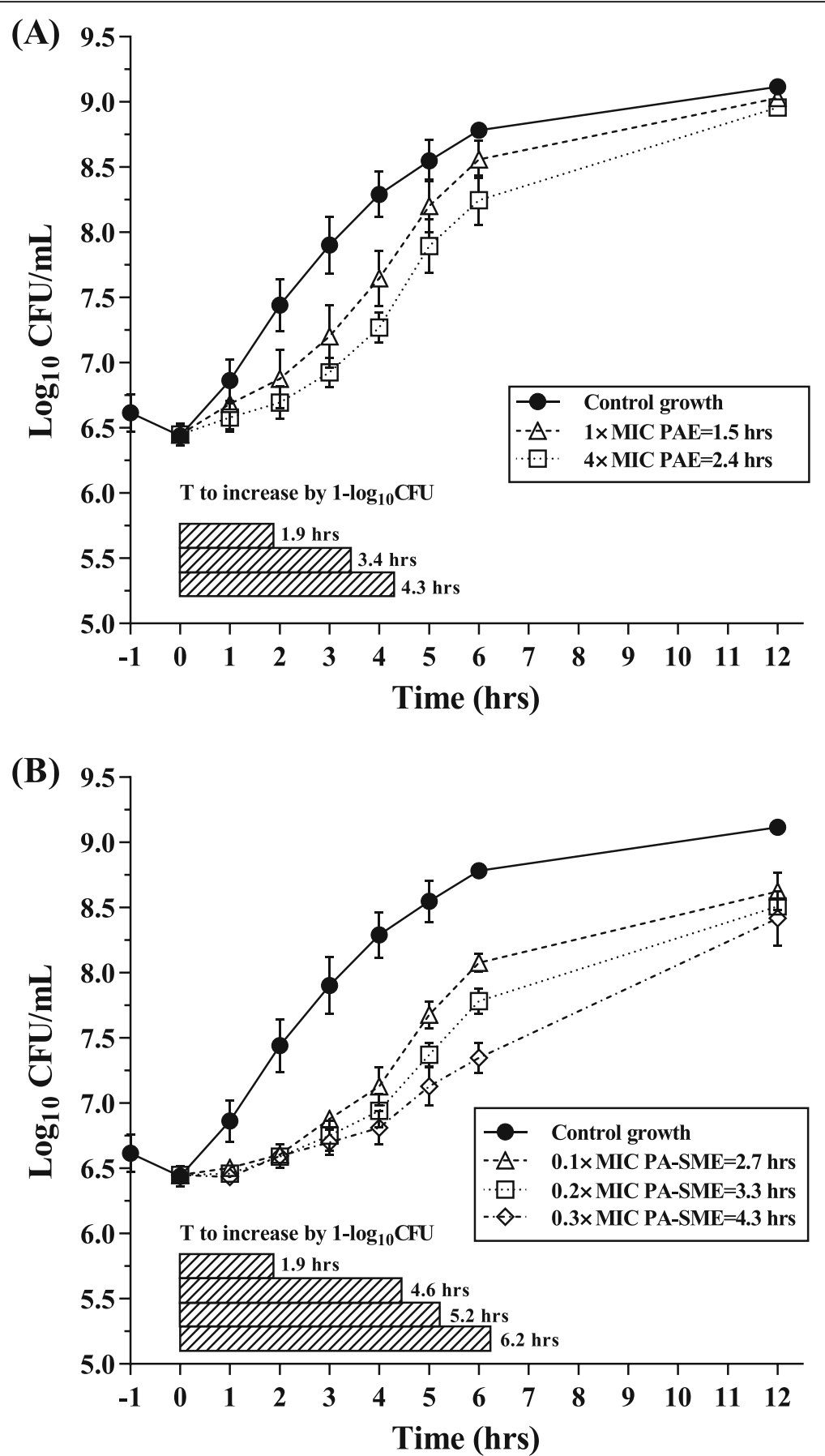

Fig. 3 In vitro PAE (a) and PA-SME (b) values for gamithromycin against H. parasuis LM15. PA-SME was determined after an initial exposure to $4 \times$ MIC. Each symbol represents the mean \pm SD of data from three independent experiments $(n=3)$. The black horizontal bars represent the time required for viable counts of bacteria to increase by $1.0 \log _{10} \mathrm{cfu} / \mathrm{mL}$ in the drug removal and sub-MIC treatment phases

to the downregulation of oprM gene (encoding an active efflux pump) and increased outer-membrane permeability in biological fluids [15]. Supporting this notion, previous studies showed markedly lower azithromycin MICs against Pseudomonas aeruginosa in eukaryotic media and biological fluids such as serum and bronchoalveolar lavage fluid, compared with the recommended CA-MHB [15]. Similar observations were also noted for the potency of tulathromycin in calf serum against $M$. haemolytica and P. multocida [11]. This suggested that measuring MICs in eukaryotic media could be easily implemented to phenotypically detect acquired resistance 

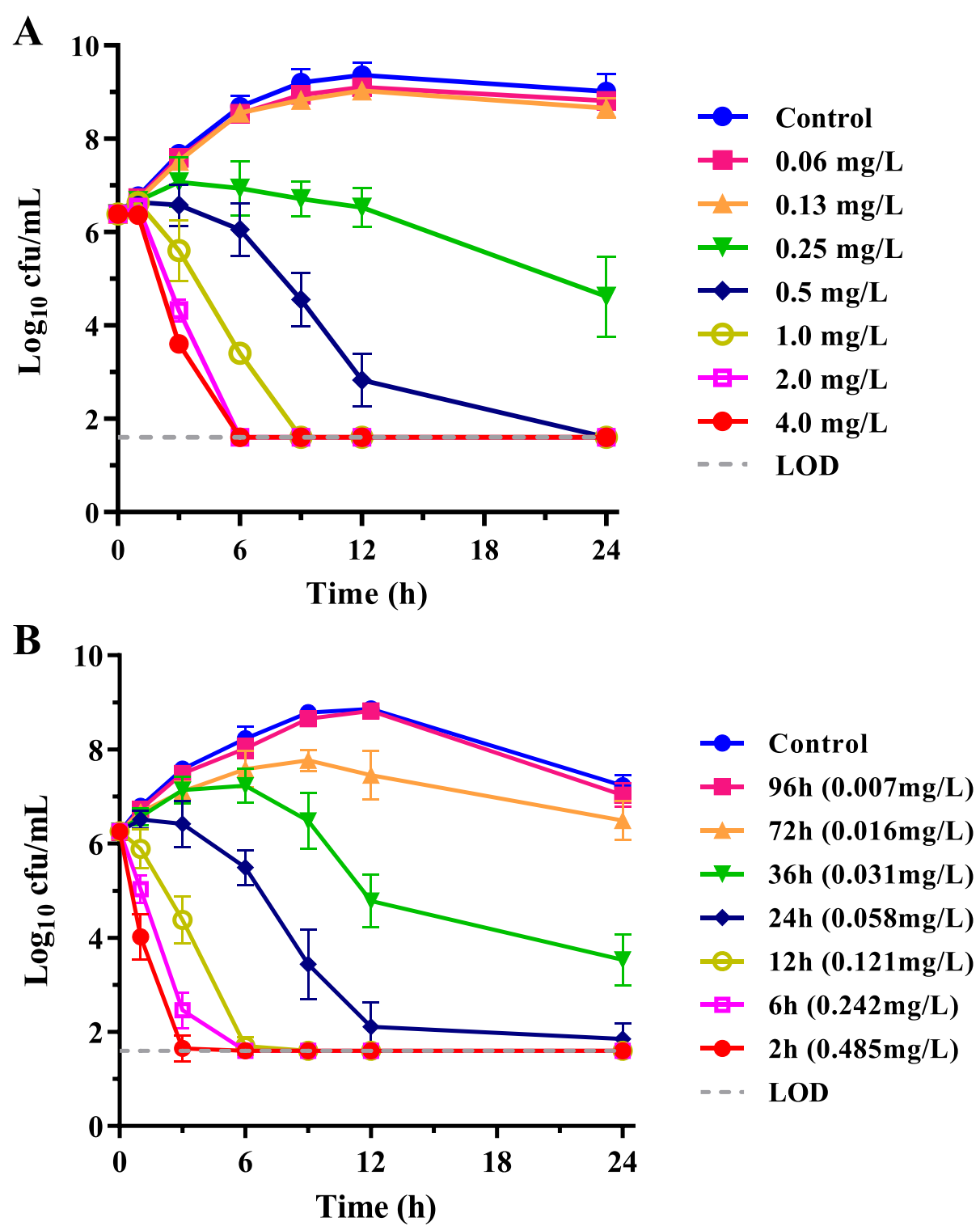

Fig. 4 In vitro and ex vivo time-kill curves of gamithromycin against $H$. parasuis LM15 in HTM broth (MIC = 0.25 mg/L in HTM; panel a) and porcine serum collected before and after IM administration of gamithromycin ( $\mathrm{MIC}=0.031 \mathrm{mg} / \mathrm{L}$ in serum; panel $\mathbf{b}$ ). Numerical values on right brackets in panel $\mathrm{B}$ are the mean concentrations of gamithromycin in serums of piglets

to macrolides [16]. In addition, the serum effects may involve specific antibody and complement activities [11, 12]. Heat-inactivation of serum resulted in a 500 -fold increase in the MIC of gamithromycin against Mycoplasma mycoides compared with normal serum [17]. Nevertheless, the large potentiation effect of serum would bring considerable benefit to the efficacy of gamithromycin in vivo for $H$. parasuis infections, where edema and hemorrhage from vascular leakage in affected lungs are part of the inflammatory process against respiratory diseases $[14,18]$. Therefore, compared to the artificial broth that is designed to be optimal for bacteriological growth in vitro, serum is a more clinically relevant biological fluid for establishing the PK/PD relationships to predict dose regimens.

Prediction of dose distribution for gamithromycin requires selection of a robust PK/PD surrogate and determination of PK/PD targets [19]. From the rate and extent of gamithromycin killing against $H$. parasuis, a concentrationdependent killing was observed in both HTM and porcine serum, with drug concentrations of $2 \times \mathrm{MIC}$ being sufficient to eradicate the sample of viable bacteria. Much discussion is available in the literature surrounding the selection of the proper PK/PD index to determine the optimal dose of macrolides [9, 20,21]. Contemporary thought on the longer acting injectable macrolides in veterinary 


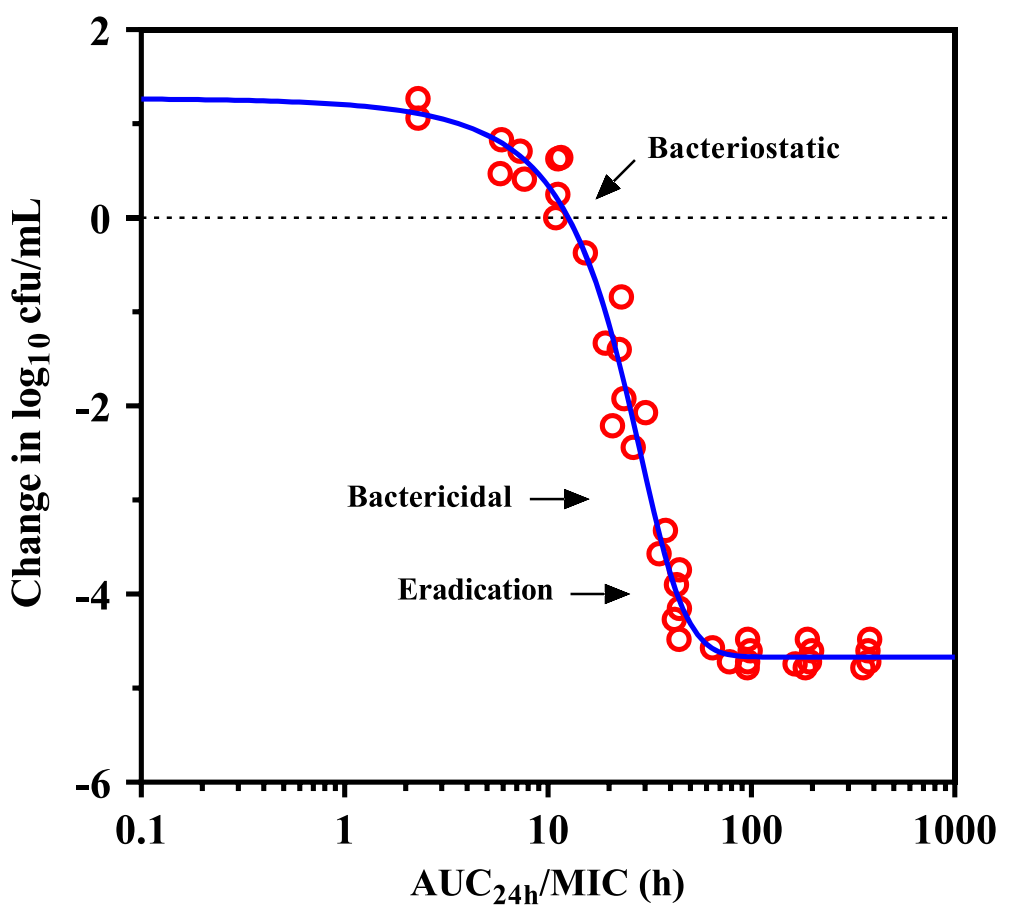

Fig. 5 Ex vivo PK/PD relationship of gamithromycin against $H$. parasuis LM15 in serums of piglets. The curve represents predicted values based on the sigmoid $E_{\max }$ equation and the individual points represent serum samples collected at time points from 0 to $96 \mathrm{~h}$

medicine is that the large predictor of efficacy is $\mathrm{AUC}_{24 \mathrm{~h}} /$ MIC ratio $[9,22]$. The $\mathrm{AUC}_{24 \mathrm{~h}} / \mathrm{MIC}$ targets for gamithromycin against $H$. parasuis associated with bactericidal and eradication effects were $30.3 \mathrm{~h}$ and $41.2 \mathrm{~h}$, respectively. This microbiological response in ex vivo PK/PD model was similar to or in excess of previously approved veterinary macrolides such as tulathromycin and tildipirosin [13, 23]. Further population dose prediction derived from Monte

Table 3 PK/PD analysis of data acquired from ex vivo time-kill experiments of gamithromycin against a representative strain of $H$. parasuis LM15 in serums collected from each piglet $(n=6)$

\begin{tabular}{|c|c|}
\hline Parameter (units) $^{a}$ & Mean $\pm S D($ min to $\max )$ \\
\hline $\mathrm{E}_{0}(\log \mathrm{cfu} / \mathrm{mL})$ & $0.91 \pm 0.13$ (0.73 to 1.05$)$ \\
\hline$E_{\max }(\log \mathrm{cfu} / \mathrm{mL})$ & $-4.68 \pm 0.06(-4.78$ to -4.61$)$ \\
\hline$E_{\max }-E_{0}(\log c f u / m L)$ & $-5.59 \pm 0.15(-5.74$ to -5.39$)$ \\
\hline$E C_{50}(h)$ & $24.3 \pm 3.88(19.5$ to 29.9$)$ \\
\hline Slope (N) & $4.37 \pm 2.12$ (2.69 to 8.34$)$ \\
\hline $\mathrm{AUC}_{24 \mathrm{~h}} / \mathrm{MIC}$ for bacteriostatic effect (h) & $15.8 \pm 4.43(12.6$ to 24.5$)$ \\
\hline $\mathrm{AUC}_{24 h} / \mathrm{MIC}$ for bactericidal effect (h) & $30.3 \pm 4.40(24.3$ to 36.4$)$ \\
\hline $\mathrm{AUC}_{24 \mathrm{~h}} / \mathrm{MIC}$ for eradication effect (h) & $41.2 \pm 7.48(32.8$ to 52.5$)$ \\
\hline
\end{tabular}

${ }^{a} \mathrm{E}_{0}$, the change in $\log _{10} \mathrm{cfu} / \mathrm{mL}$ after $24 \mathrm{~h}$ of incubation in the no drug control sample; $E_{\max }$ difference in greatest amount of bacterial reduction ( $\log _{10} \mathrm{cfu} /$ $\mathrm{mL}$ ); $\mathrm{EC}_{50}$ is the $\mathrm{AUC}_{24 \mathrm{~h}} / \mathrm{MIC}$ producing $50 \%$ of the maximal effect; $\mathrm{N}$, the slope of dose-response curve. The bacteriostatic, bactericidal and eradication effects were defined as no change, $3.0 \log _{10} \mathrm{cfu} / \mathrm{mL}$ and $4.0 \log _{10} \mathrm{cfu} / \mathrm{mL}$ reductions in bacterial densities
Carlo simulations indicated that the current dose of gamithromycin $(6.0 \mathrm{mg} / \mathrm{kg})$ was sufficient for treating $H$. parasuis infections, covering more than $88.9 \%$ of the MIC distribution of study clinical isolates.

CBP is used to define susceptibility and resistance. In general, the determination of CBP should take into account the ECOFF, $\mathrm{CO}_{\mathrm{PD}}$ and clinical cutoff values [24, $25]$. Under the clinically recommended dose, the gamithromycin $\mathrm{CO}_{\mathrm{PD}}$ value $(0.25 \mathrm{mg} / \mathrm{L})$ against $H$. parasuis was lower than the ECOFF value $(1.0 \mathrm{mg} / \mathrm{L})$ in HTM broth. It probably means that the current dose $(6.0 \mathrm{mg} /$ $\mathrm{kg}$ ) is a little bit too low to treat the wild-type populations. In fact, our calculated accurate dose of gamithromycin for a PTA $\geq 90 \%$ was $6.55 \mathrm{mg} / \mathrm{kg}$ in this study, although slightly larger than the current dose. However, owing to the paucity of relevant data to bridge the relationship between MIC and clinical cure, it is practically difficult to determine a clinical cutoff in veterinary medicine $[24,26]$. In this case, VetCAST will not establish a CBP dividing the wild-type MIC distributions, and the ECOFF $(1.0 \mathrm{mg} / \mathrm{L})$ will therefore be recommended as surrogate [24]. This ECOFF value for gamithromycin in HTM broth is equivalent to the CLSI recommended breakpoint for erythromycin against streptococci [27]. Although the ECOFF or PK/PD cutoff could not replace the CBP, it still provides a useful interpretative criterion to categorize the AST results of gamithromycin. 

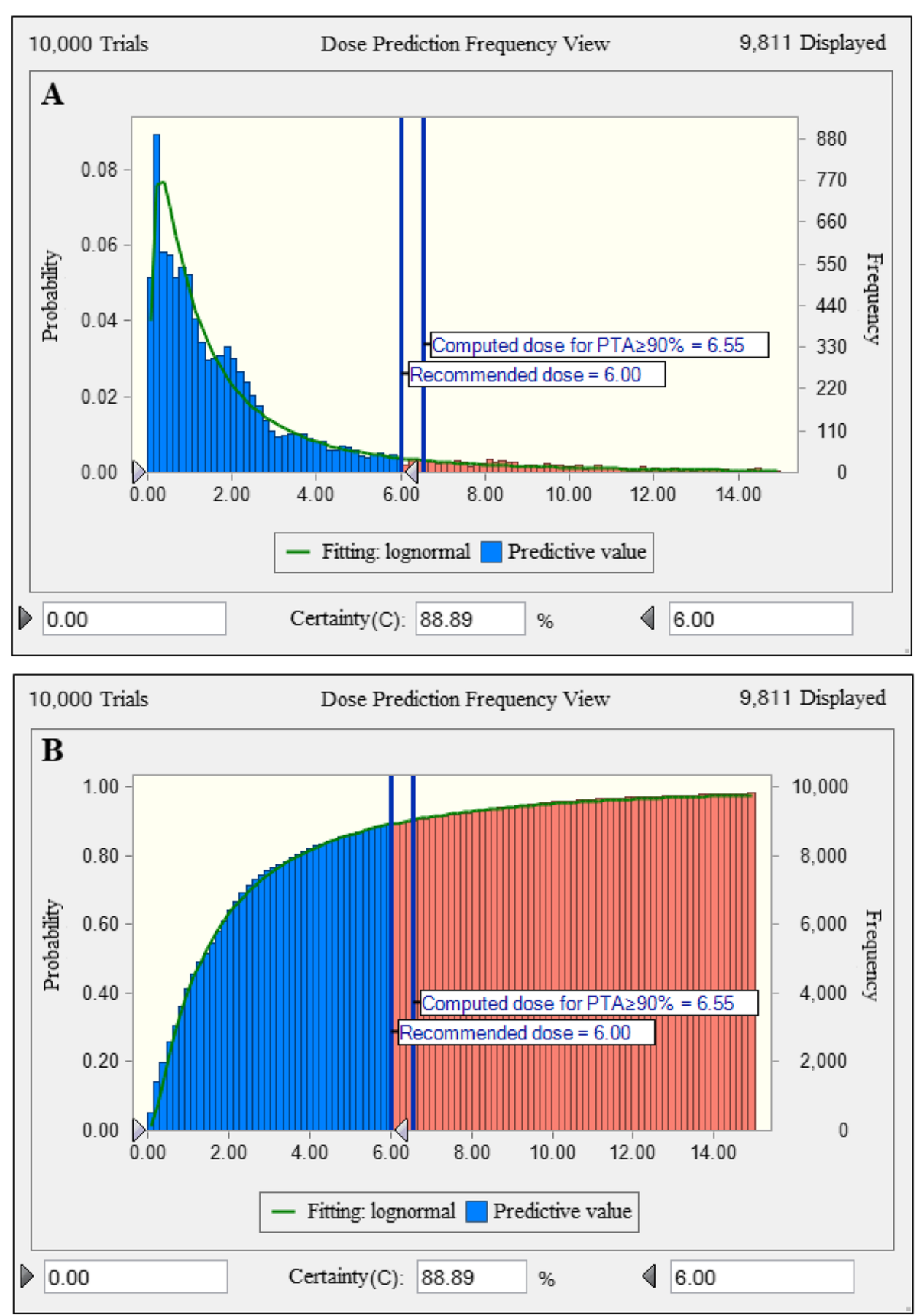

Fig. 6 Non-cumulative (a) and cumulative (b) population distributions of gamithromycin as predicted by a PK/PD model for the treatment of $H$. parasuis infection for 3 day duration in terms of PTA ( $y$-axis: 0-100\%). The vertical bars indicate the recommended dose of $6.0 \mathrm{mg} / \mathrm{kg}$ and the computed target dose for a PTA of $90 \%$. Dose $(0$ to $14 \mathrm{mg} / \mathrm{kg})$ is indicated on the $x$-axis

\section{Conclusions}

In conclusion, we have demonstrated that: (i) the matrix effects of serum on susceptibility is an important factor accounting for markedly augmented activity of gamithromycin in piglets and (ii) the current dose of $6.0 \mathrm{mg} / \mathrm{kg}$ gamithromycin was estimated to be appropriate, achieving bactericidal activity against $H$. parasuis with a PTA of $\geq 88.9 \%$. In addition, our finding is to our knowledge the first to reveal the ECOFF and PK/PD cutoff values of gamithromycin against $H$. parasuis in piglets. However, with the paucity of clinical data for gamithromycin to establish a clinical cutoff against $H$. parasuis, the ECOFF value of $1.0 \mathrm{mg} / \mathrm{L}$ will be recommended as surrogate. Nevertheless, the PK/PD-based dose prediction and cutoff determination will provide a 
Table 4 The probabilities of target attainment (PTAs) for typical $\mathrm{AUC}_{24 \mathrm{~h}} / \mathrm{MIC}$ targets at each possible MIC in HTM broth

\begin{tabular}{|c|c|c|c|c|c|c|c|}
\hline \multirow{2}{*}{$\begin{array}{l}\text { Typical } \\
\text { AUC }_{24 h} / \\
\text { MIC } \\
\text { targets } \\
\text { (h) }{ }^{a} \\
\end{array}$} & \multicolumn{7}{|c|}{ PTA (\%) at each possible MIC (mg/L) in HTM broth ${ }^{b}$} \\
\hline & 0.063 & 0.125 & 0.25 & 0.5 & 1.0 & 2.0 & 4.0 \\
\hline 15.8 & 100 & 100 & 100 & 95.9 & 31.2 & 0.78 & 0.17 \\
\hline 30.3 & 100 & 100 & 96.7 & 37.2 & 1.35 & 0.18 & 0.06 \\
\hline 41.2 & 100 & 99.5 & 85.4 & 3.31 & 0.57 & 0.12 & 0.05 \\
\hline
\end{tabular}

${ }^{a} \mathrm{AUC}_{24 \mathrm{~h}} / \mathrm{MIC}$ target required to produce bacteriostatic ( $\left.15.8 \mathrm{~h}\right)$, bactericidal $(30.3 \mathrm{~h})$ and eradication $(41.2 \mathrm{~h})$ effects in serum

${ }^{b}$ The scaling factor that bridged the MICs between HTM and serum has been taken into account when calculating the PTA

framework for further optimization of gamithromycin dosing regimens and for resistance surveillance.

\section{Methods}

\section{Antibiotics and bacterial strains}

Analytical-grade gamithromycin powder was obtained from NMT Biotech (Jiangsu, China) and reconstituted according to the manufacturer's recommendations. Gamithromycin injectable solution (Zactran $150 \mathrm{mg} / \mathrm{mL}$ ) used for PK studies was purchased commercially from Merial Animal Health Ltd., 31,000 Toulouse, France.

We isolated 192 clinical $H$. parasuis strains from heart, lung, brain, peritoneum, pericardial sac and joint fluids of diseased swine suffering polyserositis, pneumonia or meningitis in five different provinces of China in 2010 to 2018. A well-characterized representative strain of $H$. parasuis LM15 (serotype 5) was used for ex vivo timekill experiments and PK/PD modeling because serotype 5 is the primary serotype found in China [3, 28, 29]. Species identification was performed by using MALDI-TOF mass systems (Axima-Assurance-Shimadzu) as previously described [30]. H. parasuis strains were cultured with Haemophilus test medium (HTM; Becton Dickinson, Sparks, MD) broth and agar containing $15 \mu \mathrm{g} / \mathrm{mL}$ $\beta$-NAD and $5 \%$ porcine hematin.

\section{Experimental design and sample collection}

Six healthy castrated crossbred piglets (Duroc $\times$ LandracexYorkshire) weighing 14.5 to $18.6 \mathrm{~kg}(\sim 1.5$ to 2.0 months of age) were purchased commercially from the Guangzhou Fine Breed Swine Company (Guangzhou, China). Gamithromycin was administered intravenously (auricular vein), intramuscularly (femoral muscle) and subcutaneously (flank region) at a single dose of $6.0 \mathrm{mg} / \mathrm{kg}$ in accordance with a 3-treatment, 3-period randomized Latin square design. A 7-day washout period was allowed between administrations by each route. The piglets were maintained in accordance with the National Standards for Laboratory Animals of China (GB 14925-2010), and allowed ad libitum access to water and antibiotic-free food. The euthanasia procedure was carried out by pentobarbital sodium with intravenous injection when study was finished. All animal experiment procedures were approved by the Guangdong Association for Science and Technology [SYXK (Guangdong) 2019-0136] and the Institutional Animal Ethical Committee of South China Agricultural University (SCAU 2018A014).

Blood samples $(5 \mathrm{~mL})$ were collected from the jugular veins into vacutainers without anticoagulant before $(0 \mathrm{~h})$ and at $0.083,0.25,0.5,0.75,1,2,4,6,8,10,12,24,36$, 48,72 and $96 \mathrm{~h}$ after administration of gamithromycin. Serum was separated by centrifugation of blood samples at $3000 \times \mathrm{g}$ for $10 \mathrm{~min}$ and stored at $-80^{\circ} \mathrm{C}$ until analysis.

Measurement of gamithromycin in serum and PK analysis Gamithromycin concentrations in serum were determined by a validated HPLC-MS/MS method using an Agilent 1200 HPLC system linked to an API 4000 triple quadrupole mass spectrometer as previously reported $[8,31]$. In brief, serum samples $(0.5 \mathrm{~mL})$ were mixed with $0.5 \mathrm{~mL}$ of acetonitrile, followed by vortex and centrifugation at $12,000 \times \mathrm{g}$ for $10 \mathrm{~min}$. The resulting supernatant was filtered through a $0.22 \mu \mathrm{m}$ nylon syringe filter. Matrix matched calibration standards gave linear responses from 0.001 to $0.5 \mathrm{mg} / \mathrm{L}$ $\left(\mathrm{R}^{2}>0.996\right)$, with limits of quantification (LOQ) of 0.0005 $\mathrm{mg} / \mathrm{L}$. All samples with drug levels $>0.5 \mathrm{mg} / \mathrm{L}$ were diluted proportionally with control serum prior to extraction with acetonitrile. Recoveries of gamithromycin from serum ranged from 95.9 to $106.2 \%$, and both the intraday and interday variations were $<9.27 \%$ (data not shown).

All PK parameters were measured using a noncompartmental model in WinNonlin software Version 5.2.1 (Pharsight, St. Louis, MO, USA). The absolute bioavailability (F) of gamithromycin in piglets was calculated from the following equation [21, 32]:

$$
\mathrm{F} \%=\frac{\mathrm{AUC}_{0-\infty \mathrm{IM}} \text { or } \mathrm{SC}}{\mathrm{AUC}_{0-\infty \mathrm{IV}}} \times 100 \%
$$

\section{In vitro susceptibility testing, ECOFF and PAE determinations}

The MICs of gamithromycin against 192 clinical $H$. parasuis isolates were determined using broth dilution in accordance with CLSI guidelines [27]. Escherichia coli ATCC 25922 served as the quality control strain. The composition of serum and HTM broth differs significantly in electrolytes and albumin concentrations [11]. To investigate the effect of serum matrix on gamithromycin susceptibility, further $\mathrm{MIC}$ and MBC determinations were undertaken for 19 selected $H$. parasuis isolates in both porcine serum and HTM broth. The $19 \mathrm{H}$. parasuis isolates were selected to cover five different provinces of China and the whole range of gamithromycin MICs. The MBC was determined using the 
spot-plate technique to achieve a $3.0 \log _{10}$ decrease in the inoculum counts $[11,13]$.

The epidemiological cutoff [ECOFF; synonym of wildtype cutoff $\left.\left(\mathrm{CO}_{\mathrm{WT}}\right)\right]$ was the MIC that best describes the end of the wild-type distribution [33]. The ECOFF value for gamithromycin against $H$. parasuis was obtained using $\log _{2}$-transformed MIC distributions in HTM broth that was subjected to statistical goodness-of-fit and nonlinear least squares regression tests as previously described [34]. The mean and standard deviation (SD) of the normal distribution for optimum nonlinear least squares regression fitting of the MICs were determined using GraphPad Prism software (Version 8.0). The final ECOFF was measured as that MIC which captured at least $95 \%$ of the optimum MIC distributions using the ECOFFinder program [33].

The PAE and PA-SME were determined using a spectrophotometric method as described elsewhere [35]. The optical density was converted into bacterial counts by comparing to a standard curve (Figure S1). The PAE and PA-SME were defined as follows: PAE or PA-SME $=\mathrm{T}$ or $\mathrm{T}_{\mathrm{PA}}-\mathrm{C}$, where $\mathrm{T}$ or $\mathrm{T}_{\mathrm{PA}}$ is the time required for bacteria to increase by $1.0 \log _{10} \mathrm{cfu} / \mathrm{mL}$ in drug removal and sub-MIC treated phases, respectively. $\mathrm{C}$ is the corresponding time for untreated control [13].

\section{In vitro and ex vivo antimicrobial activities and PK/PD modeling}

In vitro time-kill experiments of gamithromycin against $H$. parasuis LM15 were performed using HTM broth with an initial inoculum of $10^{6} \mathrm{cfu} / \mathrm{mL}$ and 0.25 to $16 \times$ MICs of gamithromycin. Ex vivo time-kill curves were established using $0.22 \mu \mathrm{m}$ filtered porcine serum collected at specified time points from 0 to $96 \mathrm{~h}$ after IM dosing using a $10^{6} \mathrm{cfu} /$ $\mathrm{mL}$ inoculum. The cultures were incubated for $1,3,6,9,12$ and $24 \mathrm{~h}$ at $37^{\circ} \mathrm{C}$, and 10 -fold serial dilutions of samples were plated on HTM agar for viable colony counts. The limit of detection (LOD) was $40 \mathrm{cfu} / \mathrm{mL}$. The ex vivo antimicrobial effect (E) at a given gamithromycin concentration was expressed as the change in $\log _{10} \mathrm{cfu} / \mathrm{mL}$ after $24 \mathrm{~h}$ of incubation. $\mathrm{AUC}_{24 \mathrm{~h}} / \mathrm{MIC}$ ratios were calculated at each gamithromycin concentration tested. Relationships between $\mathrm{AUC}_{24 \mathrm{~h}} / \mathrm{MIC}$ and the ex vivo antimicrobial effect was estimated using the sigmoid $\mathrm{E}_{\max }$ model: $\mathrm{E}=\mathrm{E}_{0}+\left(\mathrm{E}_{\max }-\mathrm{E}_{0}\right) \times$ $\mathrm{C}^{\mathrm{N}} /\left(\mathrm{EC}_{50}{ }^{\mathrm{N}}+\mathrm{C}^{\mathrm{N}}\right)$, where $\mathrm{E}_{0}$ is the bacterial growth in drug-free sample, $\mathrm{C}$ is the $\mathrm{PK} / \mathrm{PD}$ index being examined $\left(\mathrm{AUC}_{24 \mathrm{~h}} / \mathrm{MIC}\right), \mathrm{E}_{\max }$ is the greatest amount of bacterial reduction $\left(\log _{10} \mathrm{cfu} / \mathrm{mL}\right), \mathrm{EC}_{50}$ is the $\mathrm{AUC}_{24 \mathrm{~h}} / \mathrm{MIC}$ target achieving $50 \%$ of maximal effect $\left(E_{\max }\right)$, and $\mathrm{N}$ is a sigmoid factor that describes the slope of the curve [35]. Nonlinear regression analysis was performed by WinNonlin software. The $\mathrm{AUC}_{24 \mathrm{~h}} / \mathrm{MIC}$ targets in serum required to produce bacteriostatic $(E=0)$, bactericidal $(E=-3)$ and eradication $(\mathrm{E}=-4)$ effect were calculated.

\section{Dose regimen assessment}

To investigate whether the current dose of gamithromycin $(6.0 \mathrm{mg} / \mathrm{kg})$ was adequate to cover the overall MICs of $H$. parasuis population, a dose distribution prediction was simulated using Monte Carlo simulations $(n=10$, 000) with Crystal Ball software (Oracle, Redwood City. CA, USA). The dose equation was as follows [22]:

$$
\text { Dose }(\text { for } 3 \text { days })=\frac{\mathrm{Cl}_{\text {for } 3 \text { days }} \times \mathrm{SF} \times \mathrm{MIC}_{\text {distribution }}}{f u \times \mathrm{F}}
$$

Where $\mathrm{Cl}$ is the body clearance to cover at least 3 days ( $\mathrm{L} / \mathrm{kg} / 3$ day); SF is a scaling factor obtained by dividing the target value of PK/PD index, in our case, the target $\mathrm{AUC}_{24 \mathrm{~h}} / \mathrm{MIC}$ value of 30.3 is equivalent to consider that a bactericidal effect can be obtained with a serum concentration equal to $30.3 / 24=1.26$ folds the MIC (SF = 1.26 for bactericidal effect); MIC distribution in HTM broth was divided by a scaling factor of 8.93 to bridge HTM and serum; $f u$ is the free drug fraction using a binding rate of $23 \%$ in porcine serum [21]; $\mathrm{F}$ is the bioavailability of IM dosing. In clinical practice, only susceptible $H$. parasuis isolates can be successfully treated with gamithromycin and this should be considered when calculating a dose. Therefore, for dose distribution prediction, $H$. parasuis isolates with the MICs of > ECOFF value were removed from consideration.

\section{PK/PD cutoff determination}

A 10,000-subject Monte Carlo simulation was used to calculate the $\mathrm{CO}_{\mathrm{PD}}$ value of gamithromycin against $H$. parasuis based on PK parameters, each possible MIC in HTM broth and the target $\mathrm{AUC}_{24 \mathrm{~h}} / \mathrm{MIC}$ ratios for achieving a bactericidal effect. The $\mathrm{AUC}_{24 \mathrm{~h}} / \mathrm{MIC}$ was calculated as follows [35]: $\mathrm{AUC}_{24 \mathrm{~h}} / \mathrm{MIC}=\mathrm{Dose} /[\mathrm{Cl} \times($ $\left.\left.\mathrm{MIC}_{\mathrm{HTM}} / \mathrm{SF}\right)\right]$. All PK parameters were assumed to be normally distributed. SF is a scaling factor of 8.93 to bridge the MICs between HTM and serum. The $\mathrm{CO}_{\mathrm{PD}}$ was defined as the MIC in HTM broth at which the PTA for bactericidal effect reached 90\% [26, 29].

\section{Supplementary information}

Supplementary information accompanies this paper at https://doi.org/10. 1186/s12917-020-02300-y.

Additional file 1: Figure S1. Standard curve constructed by regression of the viable bacterial counts and optical density $\left(\mathrm{OD}_{600 \mathrm{~nm}}\right)$ of $\mathrm{H}$. parasuis cultured in liquid medium. The solid points represent the observed data and the line represents the best fitting curve as follows: $y=0.7138 \ln (x)$ $+10.722\left(R^{2}=0.9857\right)$.

\section{Abbreviations}

AST: Antimicrobial susceptibility testing; AUC/MIC: The area under the concentration-time curve to MIC ratio; CBP: Clinical breakpoint; $\mathrm{CO}_{\mathrm{PD}}$ : PK/PD cutoff value (named by EUCAST as the PK/PD breakpoint); ECOFF: Epidemiological cutoff value [synonym of wild-type cutoff value $\left(\mathrm{CO}_{W T}\right)$ ]; EUCAST: European Committee on AST; MBC: Minimal bactericidal 
concentration; MIC: Minimal inhibitory concentration; PAE: Post-antibiotic effect; PA-SME: Post-antibiotic sub-MIC effect; PK/PD: Pharmacokinetic/ pharmacodynamic; PTA: Probability of target attainment; VetCAST: Veterinary Committee for AST, a subcommittee of EUCAST

\section{Acknowledgements}

Not applicable.

\section{Authors' contributions}

XPL and YFZ conceived of the experiments. YFZ, MXB and PL carried out the experiments. JS and YHL contributed to the data analysis. All authors read and approved the final manuscript.

\section{Funding}

This work was jointly supported by the National Natural Science Foundation of China (grant No. 31902318), the National Key Research and Development Program of China (grant No. 2016YFD0501300), the Program for Innovative Research Team in the University of Ministry of Education of China (grant No. IRT_17R39), and the Foundation for Innovation and Strengthening School Project of Guangdong, China (grant No. 2016KCXTD010).

\section{Availability of data and materials}

The datasets used and/or analyzed in this study are available from the corresponding author on reasonable request.

\section{Ethics approval and consent to participate}

All animal studies were carried out in accordance with the recommendations of ethical guidelines of South China Agricultural University (SCAU). The protocol of in vivo experiment and strain isolation procedures from animals were approved by the SCAU Institutional Animal Ethics Committee (SCAU 2018A014). Individual written informed consent for the use of samples was obtained from all animal owners.

\section{Consent for publication}

Not applicable.

\section{Competing interests}

The authors declare that they have no competing interests.

\section{Author details}

${ }^{1}$ National Risk Assessment Laboratory for Antimicrobial Resistance of Animal Original Bacteria, College of Veterinary Medicine, South China Agricultural University, Guangzhou, China. ${ }^{2}$ Guangdong Provincial Key Laboratory of Veterinary Pharmaceutics Development and Safety Evaluation, South China Agricultural University, Guangzhou, China. ${ }^{3}$ Laboratory of Veterinary Pharmacology, College of Veterinary Medicine, South China Agricultural University, Guangzhou 510642, China.

Received: 18 October 2019 Accepted: 25 February 2020

Published online: 05 March 2020

\section{References}

1. Ye C, Li R, Xu L, Qiu Y, Fu S, Liu Y, Wu Z, Hou Y, Hu CA. Effects of Baicalin on piglet monocytes involving PKC-MAPK signaling pathways induced by Haemophilus parasuis. BMC Vet Res. 2019;15:98.

2. Liu S, Li W, Wang Y, Gu C, Liu X, Charreyre C, Fan S, He Q. Coinfection with Haemophilus parasuis serovar 4 increases the virulence of porcine circovirus type 2 in piglets. Virol J. 2017;14:227.

3. Jia A, Zhou R, Fan H, Yang K, Zhang J, Xu Y, Wang G, Liao M. Development of serotype-specific PCR assays for typing of Haemophilus parasuis isolates circulating in southern China. J Clin Microbiol. 2017;55:3249-57.

4. Kielstein P, Rapp-Gabrielson VJ. Designation of 15 serovars of Haemophilus parasuis on the basis of immunodiffusion using heat-stable antigen extracts J Clin Microbiol. 1992:30:862-5.

5. Ma L, Wang L, Chu Y, Li X, Cui Y, Chen S, Zhou J, Li C, Lu Z, Liu J, et al. Characterization of Chinese Haemophilus parasuis isolates by traditional serotyping and molecular serotyping methods. PLoS One. 2016;11: e0168903.

6. McCaig WD, Loving CL, Hughes HR, Brockmeier SL. Characterization and vaccine potential of outer membrane vesicles produced by Haemophilus parasuis. PLoS One. 2016;11:e0149132.
7. EMA. CVMP assessment report for ZACTRAN for pigs (EMEAN/C/000129/X/ 0027). In: The European Agency for the Evaluation of Medicinal Products. European Medicines Agency. 2015. https://www.ema.europa.eu/en/ documents/variation-report/zactran-v-c-129-x-0027-epar-assessment-reportvariation_en.pdf. Accessed 1 Mar 2020.

8. Huang RA, Letendre LT, Banav N, Fischer J, Somerville B. Pharmacokinetics of gamithromycin in cattle with comparison of plasma and lung tissue concentrations and plasma antibacterial activity. J Vet Pharmacol Ther. 2010; 33:227-37.

9. DeDonder KD, Apley MD, Li M, Gehring R, Harhay DM, Lubbers BV, White BJ, Capik SF, KuKanich B, Riviere JE, et al. Pharmacokinetics and pharmacodynamics of gamithromycin in pulmonary epithelial lining fluid in naturally occurring bovine respiratory disease in multisource commingled feedlot cattle. J Vet Pharmacol Ther. 2016;39:157-66.

10. Baggott D, Casartelli A, Fraisse F, Manavella C, Marteau R, Rehbein S, Wiedemann M, Yoon S. Demonstration of the metaphylactic use of gamithromycin against bacterial pathogens associated with bovine respiratory disease in a multicentre farm trial. Vet Rec. 2011;168:241.

11. Lees P, Illambas J, Potter TJ, Pelligand L, Rycroft A, Toutain PL. A large potentiation effect of serum on the in vitro potency of tulathromycin against Mannheimia haemolytica and Pasteurella multocida. J Vet Pharmacol Ther. 2017;40:419-28.

12. Pruul $\mathrm{H}, \mathrm{McD}$ onald PJ. Potentiation of antibacterial activity of azithromycin and other macrolides by normal human serum. Antimicrob Agents Chemother. 1992;36:10-6.

13. Zhou YF, Peng HM, Bu MX, Liu YH, Sun J, Liao XP. Pharmacodynamic evaluation and PK/PD-based dose prediction of Tulathromycin: a potential new indication for Streptococcus suis infection. Front Pharmacol. 2017:8:684

14. Rose M, Menge M, Bohland C, Zschiesche E, Wilhelm C, Kilp S, Metz W, Allan M, Ropke R, Nurnberger M. Pharmacokinetics of tildipirosin in porcine plasma, lung tissue, and bronchial fluid and effects of test conditions on in vitro activity against reference strains and field isolates of Actinobacillus pleuropneumoniae. J Vet Pharmacol Ther. 2013;36:140-53.

15. Buyck JM, Plesiat P, Traore H, Vanderbist F, Tulkens PM, Van Bambeke F. Increased susceptibility of Pseudomonas aeruginosa to macrolides and ketolides in eukaryotic cell culture media and biological fluids due to decreased expression of oprM and increased outer-membrane permeability. Clin Infect Dis. 2012:55:534-42

16. Mustafa MH, Khandekar S, Tunney MM, Elborn JS, Kahl BC, Denis O, Plesiat P, Traore $\mathrm{H}$, Tulkens PM, Vanderbist F, et al. Acquired resistance to macrolides in Pseudomonas aeruginosa from cystic fibrosis patients. Eur Respir J. 2017; 49:1601847.

17. Mitchell JD, Goh S, McKellar QA, McKeever DJ. In vitro pharmacodynamics of gamithromycin against Mycoplasma mycoides subspecies mycoides small Colony. Vet J. 2013;197:806-11.

18. Zhang B, Tang C, Liao M, Yue H. Update on the pathogenesis of Haemophilus parasuis infection and virulence factors. Vet Microbiol. 2014;168:1-7.

19. Papich MG. Pharmacokinetic-pharmacodynamic (PK-PD) modeling and the rational selection of dosage regimes for the prudent use of antimicrobial drugs. Vet Microbiol. 2014;171:480-6.

20. Watteyn A, Devreese M, De Baere S, Wyns H, Plessers E, Boyen F, Haesebrouck F, De Backer P, Croubels S. Pharmacokinetic and pharmacodynamic properties of gamithromycin in Turkey poults with respect to Ornithobacterium rhinotracheale. Poult Sci. 2015;94:2066-74.

21. Wyns H, Meyer E, Plessers E, Watteyn A, De Baere S, De Backer P, Croubels S. Pharmacokinetics of gamithromycin after intravenous and subcutaneous administration in pigs. Res Vet Sci. 2014;96:160-3.

22. Toutain PL, Potter T, Pelligand L, Lacroix M, Illambas J, Lees P. Standard PK PD concepts can be applied to determine a dosage regimen for a macrolide: the case of tulathromycin in the calf. J Vet Pharmacol Ther. 2017; 40:16-27.

23. Lei Z, Liu Q, Qi Y, Yang B, Khaliq H, Xiong J, Moku GK, Ahmed S, Li K, Zhang $\mathrm{H}$, et al. Optimal regimens and cutoff evaluation of Tildipirosin against Pasteurella multocida. Front Pharmacol. 2018:9:765.

24. Toutain PL, Bousquet-Melou A, Damborg P, Ferran AA, Mevius D, Pelligand L. Veldman KT, Lees P. En Route towards European Clinical Breakpoints for Veterinary Antimicrobial Susceptibility Testing: A Position Paper Explaining the VetCAST Approach. Front Microbiol. 2017:8:2344.

25. Turnidge JD, Martinez MN. Proposed method for estimating clinical cut-off $(\mathrm{COCL})$ values: an attempt to address challenges encountered when setting clinical breakpoints for veterinary antimicrobial agents. Vet J. 2017;228:33-7. 
26. Mouton JW, Brown DF, Apfalter P, Canton R, Giske CG, Ivanova M, MacGowan AP, Rodloff A, Soussy CJ, Steinbakk M, et al. The role of pharmacokinetics/pharmacodynamics in setting clinical MIC breakpoints: the EUCAST approach. Clin Microbiol Infect. 2012;18:E37-45.

27. CLSI. Performance standards for antimicrobial disk and dilution susceptibility tests for bacteria isolated from animals: approved standard, 5th ed. CLSI document VET01. Wayne: Clinical and Laboratory Standards Institute; 2018

28. Sun J, Xiao X, Huang RJ, Yang T, Chen Y, Fang X, Huang T, Zhou YF, Liu YH. In vitro dynamic pharmacokinetic/Pharmacodynamic (PK/PD) study and COPD of Marbofloxacin against Haemophilus parasuis. BMC Vet Res. 2015;11:293.

29. Xiao X, Sun J, Chen Y, Huang RJ, Huang T, Qiao GG, Zhou YF, Liu YH. In vitro dynamic pharmacokinetic/pharmacodynamic (PK/PD) modeling and PK/PD cutoff of cefquinome against Haemophilus parasuis. BMC Vet Res. 2015;11:33.

30. Moreno LZ, Silva GF, Gomes VT, Matajira CE, Silva AP, Mesquita RE, Lotto NP, Ferreira TS, Christ AP, Sato MI, et al. Application of protein profiling of virulent Haemophilus parasuis by MALDI-TOF mass spectrometry. J Infect Dev Ctries. 2016;10:678-81.

31. Berlin S, Randow T, Scheuch E, Grube M, Venner M, Siegmund W. Pharmacokinetics and pulmonary distribution of gamithromycin after intravenous administration in foals. J Vet Pharmacol Ther. 2017:40:406-10.

32. Toutain PL, Bousquet-Melou A. Bioavailability and its assessment. J Vet Pharmacol Ther. 2004;27:455-66.

33. Turnidge J, Kahlmeter G, Kronvall G. Statistical characterisation of bacterial wild-type MIC value distributions and the determination of epidemiological cut-off values. Clin Microbiol Infect. 2006;12:418-25.

34. Tao MT, Zhou YF, Sun J, Liu YH, Liao XP. Establishment of valnemulin susceptibility breakpoint against Clostridium perfringens in rabbits. Anaerobe. 2017:48:118-20.

35. Zhou YF, Yu Y, Sun J, Tao MT, Zhou WJ, Li X, Liao XP, Liu YH. Ex vivo pharmacokinetic/pharmacodynamic relationship of valnemulin against Clostridium perfringens in plasma, the small intestinal and caecal contents of rabbits. Anaerobe. 2016;39:150-7.

\section{Publisher's Note}

Springer Nature remains neutral with regard to jurisdictional claims in published maps and institutional affiliations.

Ready to submit your research? Choose BMC and benefit from:

- fast, convenient online submission

- thorough peer review by experienced researchers in your field

- rapid publication on acceptance

- support for research data, including large and complex data types

- gold Open Access which fosters wider collaboration and increased citations

- maximum visibility for your research: over $100 \mathrm{M}$ website views per year

At $\mathrm{BMC}$, research is always in progress.

Learn more biomedcentral.com/submissions 